\title{
USER EVALUATIONS OF CONTRAST-DOMINANT DISCOMFORT GLARE IN DIM DAYLIT SCENARIOS: PRELIMINARY FINDINGS
}

\author{
Geraldine Quek ${ }^{1}$, Jan Wienold ${ }^{1}$, Marilyne Andersen ${ }^{1}$ \\ 1 École Polytechnique Fédérale de Lausanne, Lausanne, SWITZERLAND \\ geraldine.quek@epfl.ch
}

\begin{abstract}
Discomfort glare metrics typically consider at least one of the two effects of discomfort glare saturation and contrast - in their equation. The former occurs when there is an excessively bright glare source in the field of view, while the latter occurs when there is a high luminance ratio between the glare source and the adaptation level of the eye. We hypothesize that the contrast effect may dominate in low-light scenarios such as those commonly found in open-plan offices. Thus, we designed and carried out a user study in controlled laboratory conditions with 63 participants with a total of 252 scenes to investigate discomfort glare evaluations in dim daylight office environments with low adaptation levels. Our preliminary findings support our hypothesis that contrast-driven metrics predict glare responses in a more reliable way than hybrid metrics at low adaptation levels where the contrast effect dominates, which underlines the need for refining glare metrics in low brightness conditions.
\end{abstract}

Keywords: Discomfort Glare, Glare metrics, Daylight, Visual Comfort, Psychophysics

\section{Introduction}

Daylighting in buildings not only serve as an energy-saving, flicker-free source of illumination, but also has many benefits towards human health and well-being (Aries et al., 2015; Knoop et al., 2020). However, badly controlled daylighting can also bring about problems such as visual discomfort and over-heating. One way to maintain occupants' visual comfort is to reduce occurrences of discomfort glare through good daylighting design; discomfort glare prediction metrics, together with physically accurate daylighting simulations can help practitioners to make design decisions from early-design stages. Current discomfort glare metrics account for at least one of the two effects of glare; saturation and contrast effect. The saturation effect can be described as an excessive amount of light entering the eye, thereby creating glare, while the contrast effect can be roughly described as an excessive luminance ratio between a glare source in the field of view and the overall adaptation level of the eye (which could typically occur in dim conditions). Hybrid metrics that consider both effects are found to be most robust in describing users' glare responses in most scenarios thus far (Wienold et al., 2019). However, the predictive performance of glare metrics is limited to the conditions from which they were developed. For example, since hybrid metrics like Daylight Glare Probability (DGP) were developed from user studies in bright daylit conditions, they rely heavily on the vertical illuminance term. However, DGP was found to underpredict visual discomfort in post-occupancy evaluations of deep open plan offices for instance, which are increasingly found in cities and usually come with lower vertical illuminances overall, and thus with lower adaptation levels when exposed to glare sources (Hirning et al., 2014; Jakubiec et al., 2020; Pierson et al., 2019). As part of a quest to extend discomfort glare metrics to a wider range of scenarios, we designed and conducted user studies that mimicked such low-light environments. In this paper, we report preliminary findings and behaviour of common glare metrics.

\section{Background}

As termed by Hopkinson in 1966, there are two main effects of glare: "One is a contrast effect, which results when a light source, possibly only of moderate brightness, is seen in an environment of much lower brightness and so causes glare by contrast; and the other is a saturation effect, which results when any part of the retina, even the whole retina, is stimulated by light at such a level that the maximum possible rate of neural response from the retinal elements is generated. A snow- covered landscape illuminated by full sun light is completely devoid of contrast, but most people experience acute discomfort due to the saturation of the whole visual response mechanism" (Hopkinson et al., 1966). Early glare metrics such as CIE 
Glare Index (CGI), Daylight Glare Index (DGI), Unified Glare Rating (UGR) were developed from electric lighting conditions (Einhorn, 1969; Chauvel et al., 1982; International Commission On Illumination (CIE), 1995), and, as a result, only accounted for the contrast effect of glare. The Experimental Unified Glare Rating (UGRexp) metric later attempted to combine the two effects of glare in its equation by involving average luminance $\left(\mathrm{L}_{\text {avg }}\right)$ to represent the saturation effect in addition to the original contrast term in UGR. In the same study, DGImod, a modified DGI, stays a contrast-driven glare metric, but the adaptation level includes an addition of a weighted contribution of the source luminance to the background luminance (Fisekis et al., 2003). Later on, Daylight Glare Probability (DGP) was developed through extensive user studies in daylit office environments under controlled laboratory conditions (Wienold and Christoffersen, 2006) and explicitly includes $E_{v}$ both as the saturation term and also the adaptation level in its contrast term as follows:

$$
\mathrm{DGP}=\mathrm{a} \cdot \underbrace{\mathrm{E}_{\mathrm{V}}}_{\text {Saturation term }}+\underbrace{\mathrm{b}}_{\mathrm{E} \text { ) }} \cdot \underbrace{\log 10\left(1+\sum_{\mathrm{i}=1}^{\mathrm{n}} \frac{\mathrm{L}_{\mathrm{i}}^{2} \omega_{\mathrm{i}}}{\mathrm{E}_{\mathrm{V}}^{1.87} \mathrm{P}_{\mathrm{i}}^{2}}\right)}_{\text {Contrast term (Log_gc) }}+\mathrm{c})
$$

where

$\mathrm{L}_{\mathrm{i}} \quad$ is the luminance of the glare source for the $\mathrm{i}$-th glare source

$\omega_{i} \quad$ is the solid angle of the glare source in steradians

$\mathrm{P}_{\mathrm{i}} \quad$ is the position index of the glare source

$\mathrm{E}_{\mathrm{v}} \quad$ is the vertical illuminance

$a=5.87 \cdot 10^{-5}, b=9.18 \cdot 10^{-2}$ and $c=0.16$

However, as the lighting conditions in the developmental dataset of DGP were systematically brightly lit conditions in single room offices, the model's outcomes may not correlate well with glare responses in deep open plan offices (Isoardi et al., 2012; Hirning et al., 2014, 2017). In these cases, the glare sources in the occupant's field of view usually come from façade areas somewhat far away, and the overall vertical illuminance is usually lower (Jakubiec et al., 2020; Pierson et al., 2019). Annual glare simulations in a typical open-plan office model in Geneva also showed that contrast-dominant glare scenarios occur far more frequently than saturationdominant scenarios (Quek et al., 2021a). A contrast-driven glare metric, Unified Glare Probability (UGP), which takes a similar equation to the UGR, was suggested for these openplan scenarios (Hirning et al., 2017). Recent studies also showed that while hybrid metrics, like Daylight Glare Probability (DGP), outperform other single-effect metrics (Wienold et al., 2019), contrast-driven glare metrics were found to describe discomfort glare responses better than saturation-driven ones in dim environments with lower adaptation levels (Quek et al., 2021b). Although DGP works relatively well in most scenarios, there is still room for improvement in glare prediction for scenarios outside of the range of brightly lit scenarios from which it was developed. In low-light scenarios where adaptation level of the eye is lower, we hypothesize that the contrast effect takes dominance rather than the saturation effect. To that end, we devised and carried out a user study to investigate users' discomfort glare evaluations as well as the predictive ability of glare metrics in dim daylight office settings.

\section{Methodology}

The aim of the user studies is to investigate the performance of existing glare metrics in predicting discomfort glare in the lower ranges of photopic lighting conditions commonly found in deep open-plan spaces. To this end, we designed four daylight scenes in the low photopic ranges (500-2000 lux) and conducted user studies in a controlled laboratory setup with access to daylight, mimicking an office-like workspace during the period of September-October 2020 and March-April 2021 in the DEMONA facility located in the EPFL campus in Lausanne. High dynamic range (HDR) images and other measurements were taken alongside questionnaire responses from participants. As a preliminary analysis, we find out and discuss which glare metrics can distinguish and rank the four experiment scenes according to participants' glare responses.

\subsection{Experiment design}

We designed the low-light lighting conditions to be between the "noticeable-disturbing" range of DGP (DGP < 0.34), by creating a daylit glare source from a diffuse window panel in dim 
adaptation levels. A within-subject, full-factorial experiment design with two independent variables, luminance, and size of the glare source, at two levels each, leading to four experiment scenes namely - "1-panel_high", "1-panel_low", "2-panel_high" and "2-panel_low" as exemplified in Figure 1. The four lighting scenes were achieved using a combination of $3 \mathrm{M}^{\mathrm{TM}}$ Envision Diffuser Film 3735-60 (60\% Tvis) and LEE 2991.2 Neutral density window films $(6.6 \%$ $T_{\text {vis }}$ ) on Perspex clear acrylic, as well as dark grey curtains hung flat on the walls and ceiling of the experiment room to lower overall illuminance. The dark panels on the window had a calculated visual transmission of $0.30 \%$ and the diffused panels had two levels of visual transmission, $36 \%$ and $44 \%$.

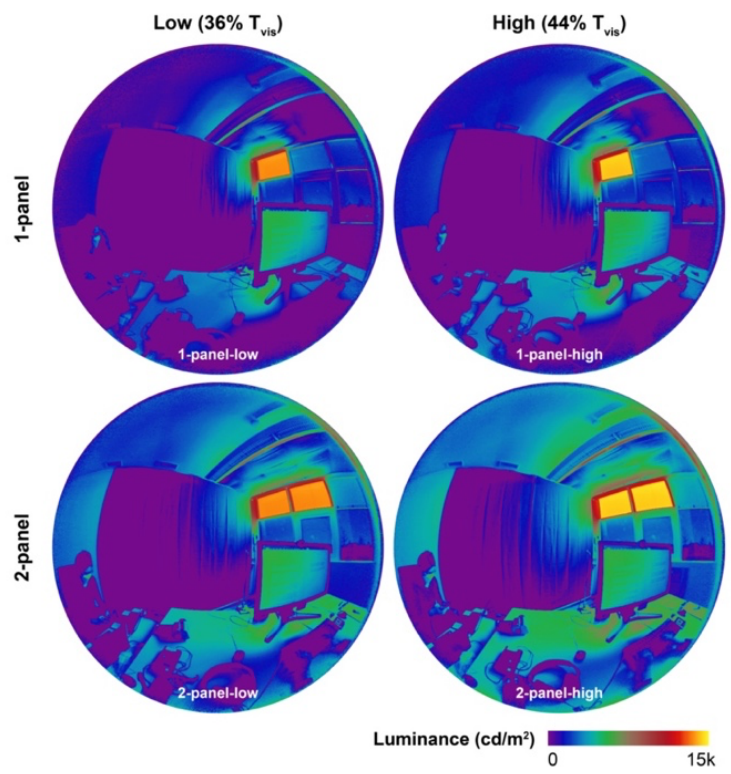

Figure 1 - Four lighting scenes using a two-level full-factorial experiment design with two variables (Luminance and size of glare source)

\subsection{Experiment protocol}

Overall, 63 human participants were recruited for the experiment, aged 18 to 30 years old in generally healthy conditions, with the age limit set to reduce the effect of aging on glare ratings. The recruited participants consisted of 20 females and 43 male gendered people, of which 31 require corrective eyewear ( 5 wearing contact lenses, 21 glasses, 5 none). After a short briefing and setting up a wearable HDR camera on their head, each participant first had to complete a baseline questionnaire on basic demographic information that was kept pseudonymized as part of the investigation design. In the 2-hour session, the four lighting conditions were then presented in a randomized order to the participant. The participants first visually acclimatized to the scene by working on a re-typing task for 10 minutes. They were then asked to complete a sampling questionnaire on the indoor environmental quality after each scenario, including answering questions on discomfort glare through a series of questionnaire items provided in Table 1 (with a definition of glare accompanying each question), and a paper-based glare indication diagram. In this diagram, participants were asked to colour the location of the glare source on the paper whenever they experienced some discomfort glare.

Table 1 - Questionnaire items on the degree of glare experienced

\begin{tabular}{|l|l|l|}
\hline Condensed name & Questionnaire item & Answer options \\
\hline "Osterhaus" & $\begin{array}{l}\text { At the moment, how would you describe glare in your } \\
\text { field of view? }\end{array}$ & $\begin{array}{l}\text { Imperceptible, Noticeable, } \\
\text { Disturbing or Intolerable }\end{array}$ \\
\hline "Binary" & $\begin{array}{l}\text { Are you experiencing any discomfort due to glare at } \\
\text { the moment? }\end{array}$ & Yes or No \\
\hline "How much" & $\begin{array}{l}\text { How much discomfort due to glare are you } \\
\text { experiencing at the moment? }\end{array}$ & $\begin{array}{l}\text { Not at all, Slightly, } \\
\text { Moderately or Very much }\end{array}$ \\
\hline
\end{tabular}




\begin{tabular}{|c|c|c|}
\hline "Interval" & $\begin{array}{l}\text { On a scale of } 0-10 \text {, how much discomfort due to glare } \\
\text { are you experiencing at the moment? }\end{array}$ & $\begin{array}{l}0 \text { (Not at all) - } 10 \text { (Very } \\
\text { much) }\end{array}$ \\
\hline "Glare diagram" & 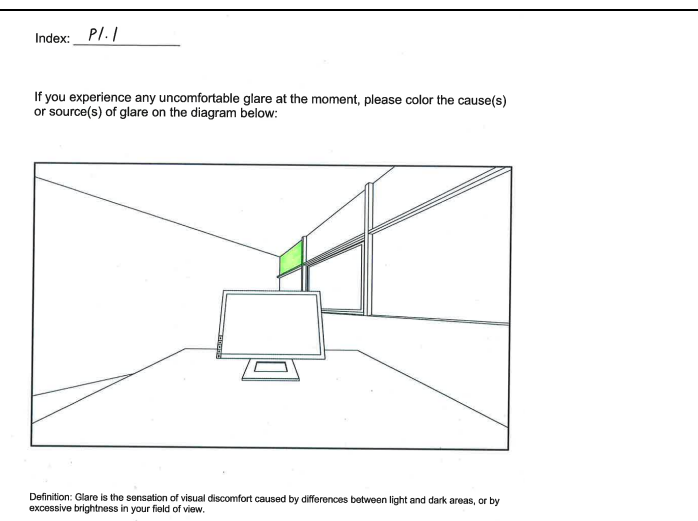 & $\begin{array}{l}\text { Color glare source or } \\
\text { Leave blank }\end{array}$ \\
\hline
\end{tabular}

This study will focus on the results of the "Osterhaus" questionnaire item as a preliminary analysis, and the results of other questionnaire items will be further investigated in a separate study. The questions are presented in a randomized order. Between each scene, the participant puts on an eye mask and listened to music through headphones to take a break while the researcher took lighting measurements and adjusted the acrylic panels for the next scene configuration.

\subsection{Measurements}

High dynamic range (HDR) images were continuously captured every 15 seconds using the LMK 98-4 color HighRes camera with a fish-eye lens (type Dörr Digital Professional DHG, equidistant projection) with a ND-2 filter (80.17 factor), and illuminance data were captured continuously using Hagner SD2 Luxmeters connected to a multi-channel amplifier MCA-1600 connected to a Keysight data acquisition system (34972A). Handheld vertical illuminance measurements were also taken using the LMT Pocketlux 2 illuminance meter, and a handheld luminance measurement on a grey card was taken using the Konica Minolta LS100 luminance meter. Spectral distribution data was also measured using the Ocean Insight Jaz spectrometer. The positions of the measurement equipment are shown on Figure 2. Other variables like thermal comfort and air quality were maintained during the experiments and were measured through indoor climate monitoring measurements on the Testo 480.

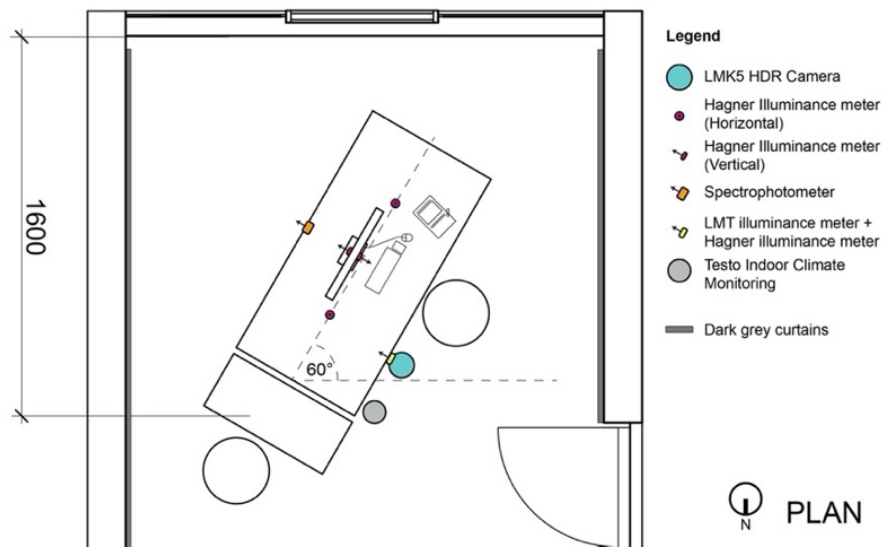

Figure 2 - Plan of experiment setup and position of measurement devices

\subsection{Processing HDR images and sorting}

The ".pf" images measured every 15 seconds in each evaluated scene using the Technoteam system were converted to ".hdr" format, resized to $800 \times 800$ pixels for faster processing, sorted 
by participant and scene, and evalglare was run on each image with the settings "-b 3000 -r 0.3 -C 0 -d" for a detailed output with the luminance threshold to detect a glare source set at $3000 \mathrm{~cd} / \mathrm{m}^{2}$ and search radius set at 0.3 radians, and low-light correction disabled. Stray glare sources (light leaks from some parts of the facade) with less than 500 pixels were re-integrated into the background luminance. To ensure that a stable sky condition throughout the typing task and questionnaire period, 18 out of 252 scenes were disqualified based on a $25 \% E_{v}$ deviation $\left(\left(\max E_{v}-\min E_{v}\right) /\right.$ mean $\left.E_{v}\right)$ threshold, resulting in a final 234 scenes evaluated by 62 unique participants, as shown in Figure 3. The HDR images were also checked for consistency with the handheld vertical illuminance measurements using the LMT Pocketlux 2 illuminance meter. The before and after Ev of each scene was averaged and compared between the HDR images and the handheld measurements, shown in Figure 4.

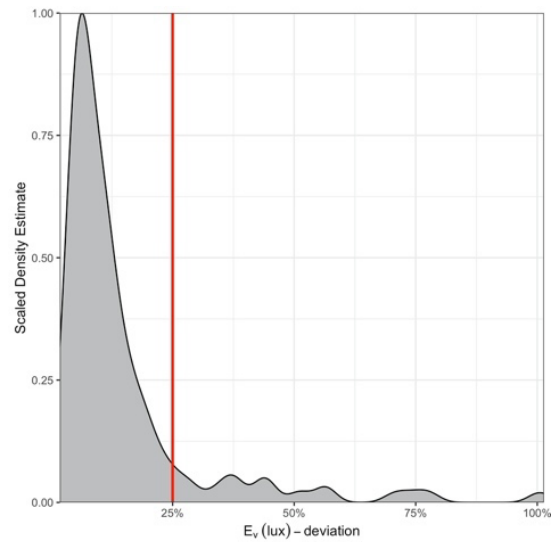

Figure 3 - Cut-off point for $E_{v}$ deviation (25\%) for sorting out stable sky conditions during timeframe of typing task and answering questionnaire

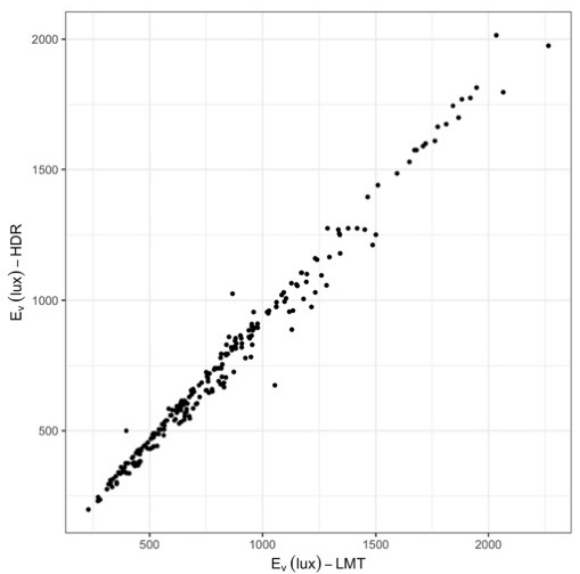

Figure 4 - Comparison between $E_{v}$ derived from the HDR images and from the handheld LMT illuminance meter (averaged first and final values)

\section{Results}

\subsection{Luminous characteristics of the four scenes}

From the processed HDR images, glare metrics, namely DGP, its two terms $E_{v}$ and Log_gc (Equation 1), UGRexp, CGI, DGI, DGI mod, UGP and UGR were then calculated from the detailed output from each image. Basic statistics of the glare metrics and its variable components were computed per scene per participant. The mean value of each variable is derived from each set of measurements during a scene (around 15-20 minutes' worth of data), and we used it as the representative value for the analysis of the evaluated scene. For brevity, data mentioned from here on refers to the representative mean value, unless otherwise stated. Figure 5 shows the distribution of the vertical illuminance $\left(E_{v}\right)$, luminance $\left(L_{s}\right)$, solid angle $\left(\omega_{i}\right)$ and position $\left(P_{i}\right)$ of the glare source of each evaluated scene. The terms usually used for representing adaptation level of the eye, $L_{b}$ and $E_{v}$, both follow a similar trend across the four scenes, with 2-panel_high 
having the highest adaptation level of all scenes. The scenes with higher luminance glare sources (1-panel_high and 2-panel_high) have a median $\mathrm{L}_{\mathrm{s}}$ of around $10500 \mathrm{~cd} / \mathrm{m}^{2}$, while the scenes with lower luminance glare sources (1-panel_low and 2-panel_low) have a median $L_{s}$ of around $7000 \mathrm{~cd} / \mathrm{m}^{2}$. The solid angle of the glare sources was distinctly different depending on 1 or 2 panel scenes, with a median 0.06 steradians for the 1-panel scenes to a median of 0.125 steradians for the 2-panel scenes. Although the position and viewing angle of the participants was fixed, the position index of the glare sources changed slightly from 3 to 4 in the 2-panel scenes. The computed glare metrics will be further described and analysed in the results section of this paper.

\subsection{Glare responses from participants}

Overall, results show that 1-panel_high scene received the highest percentage of participants who reported glare that is noticeable and above, followed by 1-panel_low, 2-panel_high, and 2panel_low. In a set of four scenes were separately evaluated by 62 unique participants, up to $25 \%$ of participants reported disturbing and above glare in one of the scenes, with $75 \%$ of participants reporting glare to be noticeable and above. This can be seen in Figure 6 where the glare responses on the "Osterhaus" questionnaire item (Osterhaus, 1996), from imperceptible to intolerable. Despite the low levels of vertical illuminance in all four scenes $\left(E_{v}<2000\right.$ lux $)$, about $50 \%$ of participants reported noticeable glare for all four scenes, while as high as $25 \%$ of participants reported glare that is disturbing and above in the 1-panel_high scenes. Although the solid angle doubled from 1-panel_high to 2-panel_high, lesser participants reported glare in 2-panel_high, which can be likely accounted to the higher adaptation level of the eye. Despite the lower luminance of 1-panel_low compared to 2-panel_high, the former received more reports of glare than the latter, which can be alluded to the fact that the general adaptation level of the eye is increased in the latter scene despite increased luminance of the glare source, leading to a reduced effect of contrast.
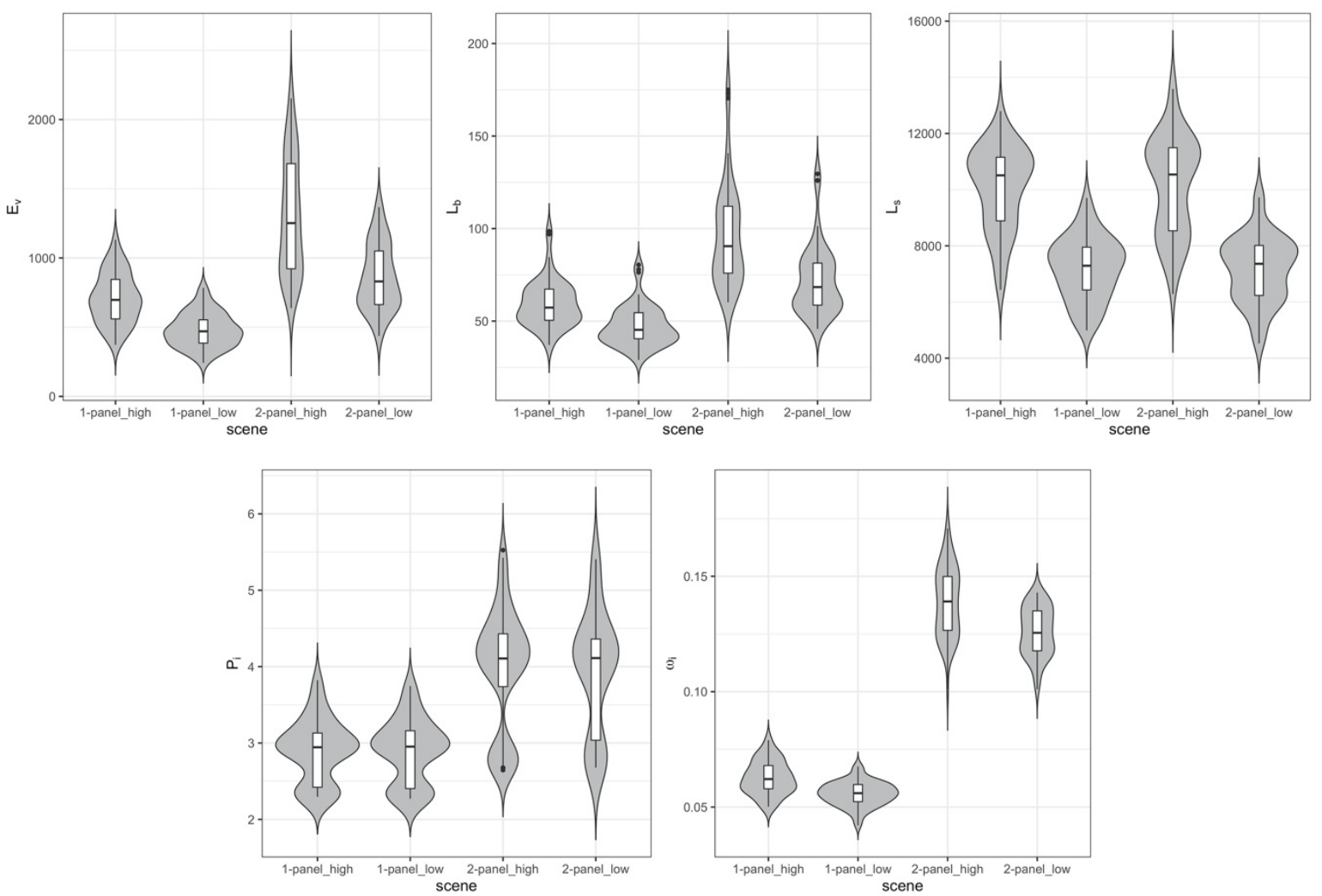

Figure 5 - Violin box plots showing distribution of mean vertical illuminance $\left(E_{v}\right)$, background luminance $\left(L_{b}\right)$, luminance $\left(L_{s}\right)$, solid angle $\left(\omega_{i}\right)$ and position index $\left(P_{i}\right)$ of the glare source across four experiment scenes 


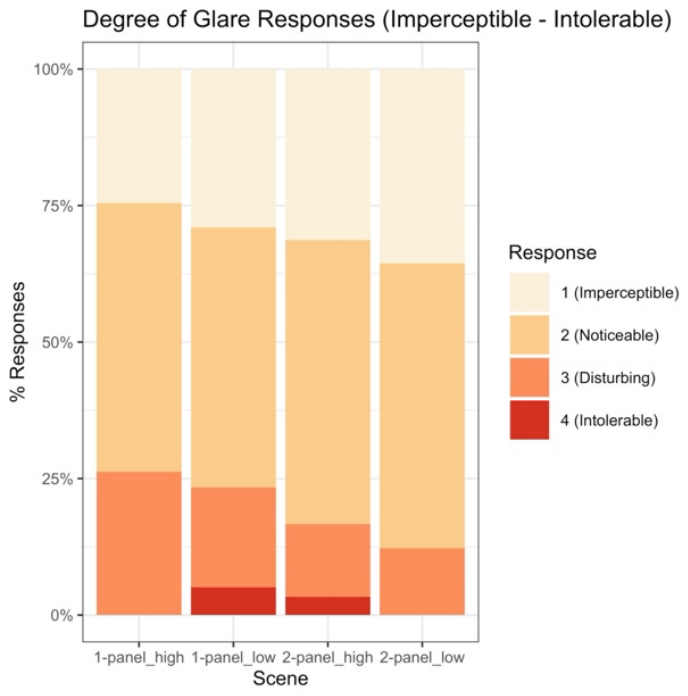

Figure 6 - Percentage of discomfort glare responses on the "Osterhaus" questionnaire item

\subsection{Comparing glare metrics vs. glare responses}

In general, contrast-driven glare metrics, DGI and DGImod, ranked all four scenes in an order consistent with that of participants (1-panel_high, 1-panel_low, 2-panel_high, 2-panel_low). Figure 7 shows the distribution of glare metrics and how they rank the four evaluated scenes. Other contrast metrics like CGI, UGR and UGP ranked three out of four scenes correctly; 2panel_high was ranked higher than 1-panel_low against the trend of collected glare responses (see Figure 8). Hybrid metrics that consider both saturation and contrast effects of glare, DGP and UGRexp both predict 2-panel_high to be ranked higher than 1-panel_high, while glare responses show otherwise (see Figure 9). The hybrid metrics clearly favour the saturation effect: a sharp spike in $E_{\vee}$ can be seen in 2-panel_high, despite the drop in Log_gc as shown in Figure 10.
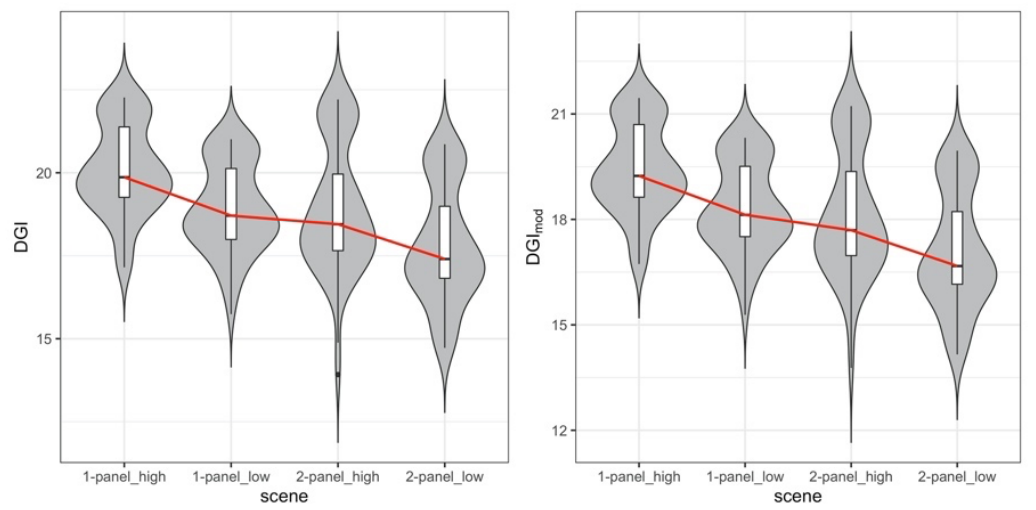

Figure 7 - Distributions and trends of contrast-driven DGI and DGI $\mathrm{mod}_{\text {across }}$ the four experiment scenes (red trendline links the median values) following the trend of glare responses 

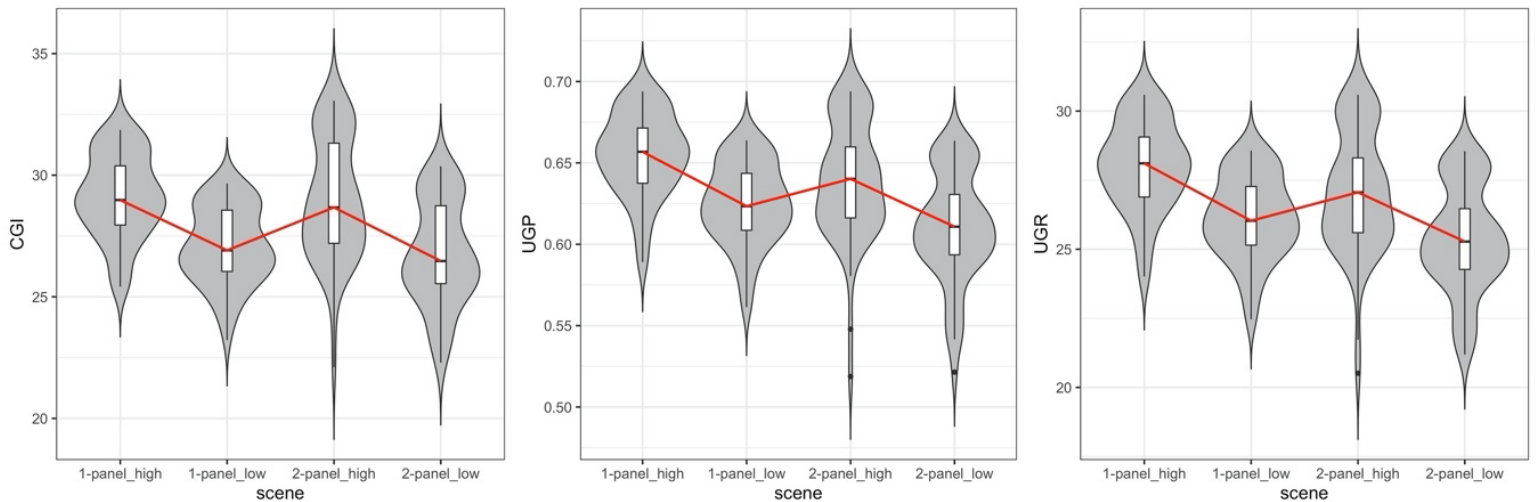

Figure 8 - Distributions and trends of other contrast-driven glare metrics across the four experiment scenes (red trendline links the median values)
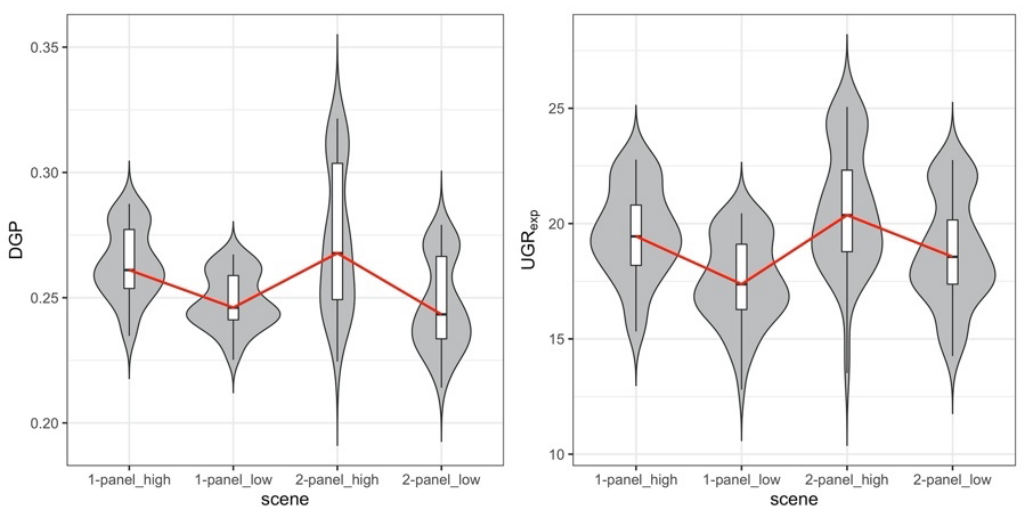

Figure 9 - Distributions and trends of "hybrid" glare metrics across the four experiment scenes (red trendline links the median values)
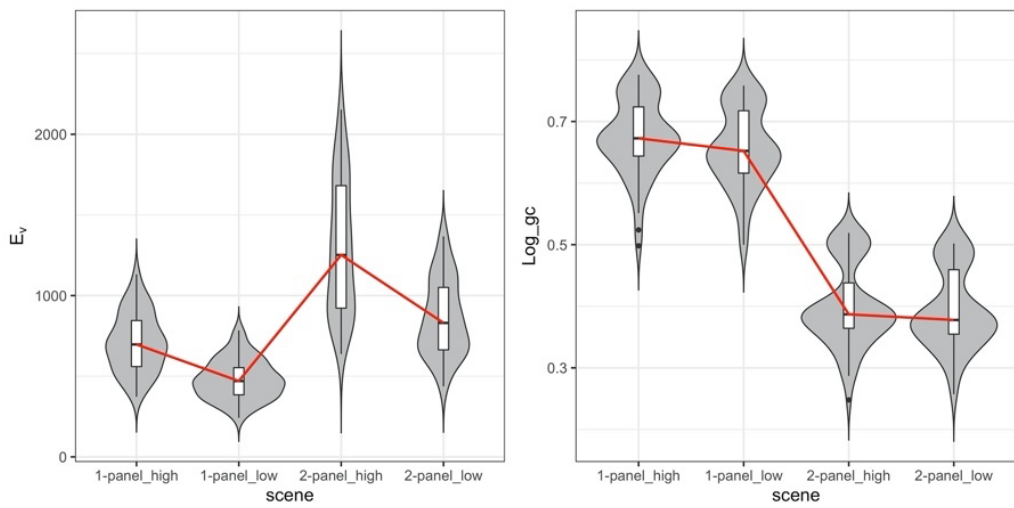

Figure 10 - Distributions and trends of the two components of DGP, $E_{v}$ (saturation effect) and Log_gc (contrast effect) across the four experiment scenes

\section{Discussion}

In this section, we examine the likely explanations for the glare measurements' behaviour in the four settings, as well as future research directions for expanding robust glare prediction to low light conditions.

As DGI and DGImod only account for the contrast effect of glare in their equations, their agreement in their ranking of the scenes to participants' responses a priori corroborates that the perception of glare in low-adaptation levels of daylight can be described primarily by the contrast effect. The adaptation level in these two metrics includes a weighted contribution of Ls and I which accounts for the fact that the eye adapts to the background luminance as well as the glare source luminance to some extent. 
Other contrast driven metrics like CGI, UGR and UGP which solely uses $L_{b}$ to represent adaptation level showed a spike in metric values, likely due to the increase in luminance and solid angle of the glare source in 2-panel_high: the adaptation level solely represented by $L_{b}$ does not seem to be appropriate, especially for such conditions where the contrast effect is the dominating effect. This preliminarily finding thus suggests that the sole usage of background luminance may be too low to indicate the increase in adaptation level of the eye, as exemplified in the behaviour of CGI, UGR and UGP versus DGI and DGImod.

On the other hand, the hybrid metrics, DGP and UGRexp, showed a spike in the 2-panel_high scene. This can be due to higher weight given to the saturation terms ( $E_{v}$ and $L_{a v g}$ respectively) in both equations. For DGP, this could be due to Log_gc given a lower overall weightage in the DGP equation, as well as that $E_{v}$ is used as the adaptation level in Log_gc, leading to a sharp decrease in the 2-panel scenes. This demonstrates the need to rethink the balance between saturation and contrast effects in hybrid metrics like the commonly used DGP, to improve glare prediction especially for low-light adaptation scenes in the photopic range. In addition, although Log_gc responds to the reduction of the contrast effect by using $E_{v}$ as the adaptation level (with an exponent of 1.87), the sharp decrease in Log_gc from 1-panel to 2-panel scenes differs to the gentle decrease of responses reporting glare. Hence, this should be further discussed in future studies.

The results signify that contrast effects are dominant in dimmer lighting conditions with lower adaptation levels. This is very important for researchers and practitioners to account for when using simplified illuminance-based metrics as they do not include contrast effects and might thus lead to misleading results. This would be the case for the simplified DGP (DGPs), where $E_{v}$ is the only variable (Wienold, 2009), or metrics like Useful Daylight Illuminance Excessive (UDle) based on horizontal illuminance thresholds. These metrics may be useful to detect saturation glare, but evidently not contrast glare. As these illuminance-based metrics are widely used in practice as they allow to reduce simulation times significantly especially when dealing with annual glare evaluations, this reservation is worth noting. However, with newer and more efficient methods of luminance map simulations, contrast-based metrics should be within reach even for annual and spatial simulations at faster and more acceptable speeds (Quek et al., 2021a; Wasilewski et al., 2021; Wasilewski, 2020).

It should be noted that while absolute glare metric threshold recommendations are outside the scope of this paper, the accuracy of glare metrics based on current thresholds might be worth looking into in future investigations. For example, all scenes were predicted to be "imperceptible" glare given the current recommended thresholds of DGP (DGP<0.34) to describe glare, although the dominating glare response was "noticeable". In addition, more discussions on glare questionnaire items are called for: although the current ranked order from the Osterhaus scale is similar to most of the other questionnaire items used in the study, results may differ, based on the questionnaire item used by the researcher. For future analysis and metric development, the HDR images should also be corrected for the displaced position $(30 \mathrm{~cm}$ on left of participant).

\section{Conclusion}

In this paper, we reported preliminary findings of discomfort glare evaluations and the trends of glare metrics from a user study that we designed and conducted in low-light daylit conditions in a controlled environment with varying luminance and size of glare source. The scene with the highest percentage of participants reporting glare was 1-panel high, followed by 1-panel low, 2-panel high, and 2-panel low. We observed whether current glare metric predictions can explain glare in the same order. Our preliminary findings show that two contrast-driven glare metrics DGI and DGImod ranked all scenes similarly to user glare responses, while other contrast-driven metrics (CGI, UGR, UGP) predicted the correct order 3 out of 4 scenes. On the contrary, hybrid metrics that consider both saturation and contrast effects (DGP and UGR exp $_{\text {) }}$ only correctly ranked 2 out of 4 scenes. The results indicate the need for further investigation into the weight of adaptation levels and saturation terms in hybrid glare metrics, especially for low-light lighting conditions commonly found in deep open plan offices. More evaluation data is thus needed to improve current glare metrics for this range of lighting conditions in detail, and research is still underway to build a larger dataset and conduct more robust statistical analyses. 


\section{References}

Aries, M., Aarts, M., van Hoof, J., 2015. Daylight and health: A review of the evidence and consequences for the built environment. Lighting Research \& Technology 47, 6-27. https://doi.org/10.1177/1477153513509258

Chauvel, P., Collins, J.B., Dogniaux, R., Longmore, J., 1982. Glare from windows: current views of the problem. Lighting Research \& Technology 14, 31-46. https://doi.org/10.1177/096032718201400103

Einhorn, H.D., 1969. A new method for the assessment of discomfort glare. Lighting Research \& Technology 1, 235-247. https://doi.org/10.1177/14771535690010040201

Fisekis, K., Davies, M., Kolokotroni, M., Langford, P., 2003. Prediction of discomfort glare from windows. Lighting Research \& Technology 35, 360-369. https://doi.org/10.1191/1365782803li095oa

Hirning, M.B., Isoardi, G.L., Cowling, I., 2014. Discomfort glare in open plan green buildings. Energy and Buildings 70, 427-440. https://doi.org/10.1016/j.enbuild.2013.11.053

Hirning, M.B., Isoardi, G.L., Garcia-Hansen, V.R., 2017. Prediction of discomfort glare from windows under tropical skies. Building and Environment, Advances in daylighting and visual comfort research 113, 107-120. https://doi.org/10.1016/j.buildenv.2016.08.005

Hopkinson, R.G., Petherbridge, P., Longmore, J., 1966. Daylighting. Heinemann.

International Commission On Illumination (CIE), 1995. Discomfort Glare in Interior Lighting (No. 117:1995).

Isoardi, G., Garcia-Hansen, V., Hirning, M., 2012. EVALUATION OF THE LUMINOUS ENVIRONMENT IN OPEN-PLAN OFFICES WITH SKYLIGHTS. Presented at the World Renewable Energy Forum (WREF 2012), Denver, Colorado, pp. 1-8.

Jakubiec, J., Quek, G., Srisamranrungruang, T., 2020. Long-term visual quality evaluations correlate with climate-based daylighting metrics in tropical offices - A field study. Lighting Research \& Technology 1477153520926528. https://doi.org/10.1177/1477153520926528

Knoop, M., Stefani, O., Bueno, B., Matusiak, B., Hobday, R., Wirz-Justice, A., Martiny, K., Kantermann, T., Aarts, M., Zemmouri, N., Appelt, S., Norton, B., 2020. Daylight: What makes the difference? Lighting Research \& Technology 52, 423-442. https://doi.org/10.1177/1477153519869758

Osterhaus, W., 1996. Discomfort glare from large area glare sources at computer workstations, in: Proceedings for the 1996 International Daylight Workshop, Building with Daylight: Energy Efficient Design. Presented at the Building with daylight: Energy efficient design, Perth, Western Australia, pp. 103-110.

Pierson, C., Sarey Khanie, M., Bodart, M., Wienold, J., 2019. DISCOMFORT GLARE CUT-OFF VALUES FROM FIELD AND LABORATORY STUDIES, in: PROCEEDINGS OF the 29th Quadrennial Session of the CIE. Presented at the Proceedings of the 29th Quadrennial Session of the CIE, International Commission on Illumination, CIE, Washington DC, USA, pp. 295-305. https://doi.org/10.25039/x46.2019.OP41

Quek, G., Wasilewski, S., Wienold, J., Andersen, M., 2021a. Spatial evaluation of potential saturation and contrast effects of discomfort glare in an open-plan office, in: BS2021. Presented at the Building Simulation 2021 Conference, Bruges, Belgium.

Quek, G., Wienold, J., Sarey Khanie, M., Erell, E., Kaftan, E., Tzempelikos, T., Konstantzos, I., Christoffersen, J., Kuhn, T., Andersen, M., 2021b. Comparing performance of discomfort glare metrics in high and low adaptation levels. Building and Environment Under Review.

Wasilewski, S., 2020. Raytraverse v1.0.4. Zenodo. https://doi.org/10.5281/zenodo.4278918

Wasilewski, S., Lars, O.G., Schregle, R., Wienold, J., Andersen, M., 2021. Raytraverse: Navigating the Lightfield to Enhance Climate-Based Daylight Modeling. Presented at the Proceedings of the Symposium on Simulation in Architecture and Urban Design 2021, Online, p. 9.

Wienold, J., 2009. Dynamic daylight glare evaluation, in: Proceedings of Building Simulation. pp. 944-951.

Wienold, J., Christoffersen, J., 2006. Evaluation methods and development of a new glare prediction model for daylight environments with the use of CCD cameras. Energy and Buildings, Special Issue on Daylighting Buildings 38, 743-757. https://doi.org/10.1016/j.enbuild.2006.03.017 
Wienold, J., Iwata, T., Sarey Khanie, M., Erell, E., Kaftan, E., Rodriguez, R., Yamin Garreton, J., Tzempelikos, T., Konstantzos, I., Christoffersen, J., Kuhn, T., Pierson, C., Andersen, M., 2019. Cross-validation and robustness of daylight glare metrics. Lighting Research \& Technology 51,983-1013. https://doi.org/10.1177/1477153519826003

\section{Appendix: Equations of Glare Metrics}

\begin{tabular}{|l|c|}
\hline CIE Glare Index (CGI): & $C G I=8 \cdot \log _{10} 2 \cdot \frac{\left[1+\frac{E_{d i r}}{500}\right]}{E_{d i r}+E_{\text {ind }}} \sum_{i=1}^{n} \frac{L_{i}^{2} \cdot \omega_{i}}{P_{i}^{2}}$ \\
\hline Daylight Glare Index (DGI): & $D G I=10 \log _{10} 0.478 \sum_{i=1}^{n} \frac{L_{i}^{1.6} \cdot \Omega_{i}^{0.8}}{L_{b}+0.07 \cdot \omega_{i}^{0.5} \cdot L_{i}}$ \\
\hline Modified Daylight Glare Index (DGI $\left.{ }_{\text {mod }}\right):$ & $D G I_{\text {mod }}=10 \log _{10} 0.478 \sum_{i=1}^{n} \frac{L_{i}^{1.6} \cdot \Omega_{i}^{0.8}}{L_{a v g}^{0.85}+0.07 \cdot \omega_{i}^{0.5} \cdot L_{i}}$ \\
\hline Unified Glare Rating (UGR): & $U G R=8 \cdot \log _{10} \frac{0.25}{L_{b}} \sum_{i=1}^{n} \frac{L_{i}^{2} \cdot \omega_{i}}{P_{i}^{2}}$ \\
\hline Experimental UGR: & $U G R_{\text {exp }}=8 \cdot \log _{10} L_{a v g}+8 \cdot \log _{10} \sum_{i=1}^{n} \frac{L_{i} \cdot \omega_{i}}{L_{b} \cdot P_{i}^{2}}$ \\
\hline Unified Glare Probability (UGP): & $U G P=\frac{1}{\left(1+\frac{2}{7}\left(\frac{1}{L_{b}} \sum_{i=1}^{n} \frac{L_{i}{ }^{2} \omega_{i}}{P_{i}^{2}}\right)^{-\frac{1}{5}}\right)^{10}}$ \\
\hline
\end{tabular}

where
$\mathrm{L}_{\mathrm{i}} \quad$ is the luminance of the $\mathrm{i}$-th glare source
$\omega_{i} \quad$ is the solid angle of the $\mathrm{i}$-th glare source
$\mathrm{P}_{\mathrm{i}} \quad$ is the Guth's position index of the i-th glare source
$L_{\mathrm{b}} \quad$ is the background luminance
$L_{\text {avg }} \quad$ is the average luminance $\left(\frac{E_{v}}{\pi}\right)$
$E_{\text {dir }} \quad$ is the direct illuminance from all glare sources
$E_{\text {ind }} \quad$ is the indirect illuminance from field of view except glare sources
$\Omega_{i} \quad$ is the corrected solid angle weighted by position index $\left(\frac{d \omega_{i}}{P_{i}^{2}}\right)$ 